\title{
Rare tumor subtypes diagnosed by molecular analysis
}

\author{
Noriomi Matsumura ${ }^{1}$
}

Published online: 8 March 2021

(C) The Japan Society of Clinical Oncology 2021

This issue contains three reports of rare tumor subtypes that have been molecularly analyzed.

Karashima et al. present two cases with a novel variant of chromophobe renal cell carcinoma showing an oncocytic phenotype [1]. Both cases were men in their late 70s. The new variant they report, unlike previous reports, showed the gain of chromosome 19. Importantly, their immunohistochemical and cytogenetic approaches allowed them to exclude oncocytoma.

Takahashi et al. report on a 75-year-old man with a primary accessory breast cancer in the right axilla [2]. Histologically, the tumor was a usual type of invasive breast cancer. The patient had a family history of breast cancer, and hereditary breast cancer was suspected. However, genetic testing of the germline did not reveal any BRCA mutations. Since the genetic risk of breast cancer due to other mutations, such as $P A L B 2$ and $A T M$, is known, multigene panel testing to identify gene mutations other than $B R C A$ may be useful.

Tanaka et al. report on a 58-year-old male patient with SMARCA4-deficient thoracic sarcoma [3]]. Driver mutations of $E G F R, A L K, R O S 1$ and $B R A F$ were not detected. A significant response was obtained with pembrolizumab in combination with carboplatin and pemetrexed.

In the future, widespread use of gene panel testing will provide a more detailed description of the molecular characteristics of rare tumor subtypes and their relationship to treatment response.

\section{References}

1. Karashima T, Kuroda N, Taguchi T et al (2020) Oncocytic variant, a novel subtype of chromophobe renal cell carcinoma: a report of two cases and a literature review. IntCancConf J. https://doi. org/10.1007/s13691-020-00459-7

2. Takahashi E, Terata K, Nanjo H et al (2021) A male with primary accessory breast carcinoma in an axilla is strongly suspected of having hereditary breast cancer. IntCancConf J. https://doi. org/10.1007/s13691-020-00466-8

3. Tanaka S, Hayashi S, Isobe Y et al (2021) Positive outcome of first-line therapy for a SMARCA4-deficient thoracic sarcomatoidtumor. IntCancConf J. https://doi.org/10.1007/s13691-021-00472 $-4$

Publisher's Note Springer Nature remains neutral with regard to jurisdictional claims in published maps and institutional affiliations.
Noriomi Matsumura

noriomi@med.kindai.ac.jp

1 Faculty of Medicine, Kindai University, Osaka-Sayama, Japan 